\title{
MULTIPLICATION OF DISTRIBUTIONS
}

\section{J. F. COLOMBEAU}

Physics often puts in evidence products which look meaningless in a mathematical sense and appear under the form of "heuristic multiplication of distributions." The most famous example is probably quantum electrodynamics founded in 1927; it was soon recognized that it led to "infinite quantities" in the form of divergent integrals from which much later (1947) finite predictions were extracted, see [4] for instance. Such products appear also in elasticity and elastoplasticity (shock waves; their importance is presently emphasized in elasticity and elastoplasticity by the need for numerical simulations of collisions), in acoustics (sound propagation in a medium with discontinuous characteristics), and in other domains, see $[1-3,10,13,14,16]$.

These examples provide motivation for mathematical attempts to define and study the multiplication of distributions. The problem is difficult since L. Schwartz (1954) proved the impossibility of a straightforward extension of the product of continuous functions, see $[8,26]$.

Strictly speaking, the reader of this text does not need to know anything about distributions in order to follow our discussion. If $\Omega$ is a nonvoid open set of the space $\mathbf{R}^{n}$ we denote by $\mathscr{C}_{0}^{\infty}(\Omega)$ or by $\mathscr{D}(\Omega)$ the space of all $\mathscr{C}^{\infty}$ functions on $\Omega$ which are null outside of a variable compact subset of $\Omega$; such functions exist: to provide an example consider the auxiliary function of one real variable $f(x)=\exp \left(1 /\left(x^{2}-1\right)\right)$ if $|x|<1, f(x)=0$ if $|x| \geq 1$. $\mathscr{C}_{0}^{\infty}(\Omega)$ is endowed with a topology and the distributions on $\Omega$ are defined as the continuous linear maps from $\mathscr{C}_{0}^{\infty}(\Omega)$ into $\mathbf{C}$. Any locally integrable function $f$ on $\Omega$ defines a distribution,

$$
\varphi \rightarrow \int_{\Omega} f(x) \varphi(x) d x, \quad \varphi \in \mathscr{C}_{0}^{\infty}(\Omega)
$$

Received by the editors October 12, 1988.

1980 Mathematics Subject Classification (1985 Revision). Primary 46F10, 35D05, 35D10. 
If $T$ is a distribution on $\Omega$ then its partial derivative $\partial T / \partial x_{1}$, $1 \leq i \leq n$, is defined by $\partial T / \partial x_{1}(\varphi)=-T\left(\partial \varphi / \partial x_{i}\right)$ which is nothing else than a formal integration by parts formula. The concept of distribution provides a convenient setting in which one can freely differentiate functions which are not derivable in the classical sense. In the sequel we shall limit ourselves to the case $\Omega=\mathbf{R}$; this is done to simplify the notation.

\section{THE ORIGIN OF OUR CONCEPT OF GENERALIZED FUNCTIONS}

The aim of this section is to sketch the original idea which led us to define a general multiplication of distributions. For this we use the nonelementary concept of differentiable functions defined on an infinite-dimensional vector space. Since we shall only sketch the idea the reader does not need to know this concept [6]; some analogy with the case of differentiable functions defined on $\mathbf{R}^{n}$ is enough. We denote by $\mathscr{E}$ or $\mathscr{C}^{\infty}$ the space of all $C^{\infty}$ functions on $\mathbf{R}$ (with a natural topology), by $\mathscr{E}^{\prime}$ the space of all linear continuous maps from $\mathscr{E}$ into $\mathbf{C}$ and by $\mathscr{D}$ the space $\mathscr{D}(\mathbf{R})$. $\mathscr{E}$ and $\mathscr{D}$ are infinite-dimensional vector spaces and have natural topologies. We denote by $\mathscr{C}^{\infty}\left(\mathscr{E}^{\prime}\right)$ and $\mathscr{C}^{\infty}(\mathscr{D})$ the respective spaces of all $C^{\infty}$ functions on $\mathscr{E}^{\prime}$ and $\mathscr{D}$. In distribution theory one proves that any element of $\mathscr{E}^{\prime}$ can be approximated (in the topology of $\mathscr{E}^{\prime}$ ) by a sequence of elements of $\mathscr{D}$; i.e. $\mathscr{D}$ is a dense subspace of $\mathscr{E}^{\prime}$. This implies that $\mathscr{C}^{\infty}\left(\mathscr{E}^{\prime}\right)$ is contained in $\mathscr{C}^{\infty}(\mathscr{D})$ through the map $\varphi \rightarrow \varphi_{\mid \mathscr{D}}$ if $\varphi \in \mathscr{C}^{\infty}\left(\mathscr{E}^{\prime}\right)$ and if $\varphi_{\mid \mathscr{D}}$ is its restriction to $\mathscr{D}$. Let $\delta_{x}$ denote the Dirac measure at the point $x \in \mathbf{R}$, i.e. $\delta_{x}(\varphi)=\varphi(x), \varphi \in \mathscr{D}$. If $\mathfrak{M}$ is the map

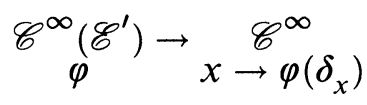

then one shows easily that the algebras $\mathscr{C}^{\infty}$ and $\mathscr{C}^{\infty}\left(\mathscr{C}^{\prime}\right) / \operatorname{Ker} \mathfrak{M}$ are isomorphic. The conclusion is that certain quotients of spaces of $C^{\infty}$ functions over certain locally convex spaces can be interpreted as very nice algebras of functions. An idea is: extend $\operatorname{Ker} \mathfrak{M}$ to an ideal $\mathscr{N}$ of $\mathscr{C}^{\infty}(\mathscr{D})$ (or of a subalgebra $\mathscr{A}$ of $\mathscr{C}^{\infty}(\mathscr{D})$ as large as possible) and try to interpret the elements of the quotient algebra

$$
\mathscr{G}=\frac{\mathscr{A}}{\mathscr{N}}
$$

as "generalized functions." This approach has been developed in $[7,8]$. Fortunately it soon became clear that one can drop the 
sophisticated concept of $C^{\infty}$ functions over $\mathscr{D}$. This gives a very elementary construction which we expose in Appendix 1. One can go on reading this text just by keeping in mind that the elements of $\mathscr{G}$ have essentially the main properties of the $C^{\infty}$ functions. We have the inclusions

$$
\mathscr{C}^{\infty} \subset \mathscr{D}^{\prime} \subset \mathscr{G} \text {. }
$$

$\mathscr{C}^{\infty}$ is a subalgebra; $\mathscr{G}$ induces on $\mathscr{D}^{\prime}$ the partial derivatives in the sense of distributions.

\section{COHERENCE With the Classical MUltiplication}

From Schwartz's impossibility result it follows that the algebra $\mathscr{C}$ of all continuous functions on $\mathbf{R}$ cannot be a subalgebra of $\mathscr{G}$. The following simple calculation shows that the algebra $\mathscr{C}_{f}$ of all piecewise continuous functions on $\mathbf{R}$ is not a subalgebra of $\mathscr{G}$. Let $Y \in \mathscr{C}_{f}$ be the Heaviside function $(Y(x)=0$ if $x<0$, $Y(x)=1$ if $x>0)$. In the algebra $\mathscr{C}_{f}$ one has if $n=2,3, \ldots$

$$
Y^{n}=Y \text {. }
$$

Thus by differentiation

$$
Y^{n-1} Y^{\prime}=\frac{1}{n} Y^{\prime}
$$

Multiplication by $Y$ gives

$$
Y^{n} Y^{\prime}=\frac{1}{n} Y Y^{\prime}
$$

Use of (2) gives

$$
\frac{1}{n+1} Y^{\prime}=\frac{1}{2 n} Y^{\prime}
$$

which is absurd ( $Y^{\prime}$ is the Dirac delta mass). The study of a shock wave with an elastic-plastic phase transition shows that in physics one needs several different Heaviside functions: they are identical to 0 for $x<0$, to 1 for $x>0$ and differ by their "microscopic" behavior at the point 0 , see $[3,10,14]$. Therefore we interpret the absurd result (3) as a consequence of (1), which should be replaced by

$$
Y^{n} \neq Y \quad \text { if } n \neq 1 .
$$

The classical product in $\mathscr{C}_{f}$, i.e. (1), cannot be used for calculations involving multiplications of distributions. Indeed in $\mathscr{G}$ one has $\left(1^{\prime}\right)$. The algebra $\mathscr{C}_{f}$ is not a subalgebra of $\mathscr{G}$. Schwartz's 
result has been interpreted negatively; for us the same fact is interpreted positively: since physics imposes the need for several different Heaviside functions it is very fortunate that mathematics leads to the same conclusion.

It is fortunate also that the classical product in $\mathscr{C}_{f}$ is very close to the new product in $\mathscr{G}:$ in order to formulate this we introduce the concept of "association": an element $G$ of $\mathscr{G}$ is said to be associated with 0 iff for any $\psi \in \mathscr{C}_{0}^{\infty}(\Omega)$ the integral

$$
\int_{\mathbf{R}} G(x) \psi(x) d x
$$

is null in a natural sense, see Appendix 1.

We say that $G_{1}$ and $G_{2} \in \mathscr{G}$ are associated with each other iff $G_{1}-G_{2}$ is associated with 0 ; we write $G_{1} \approx G_{2}$. One proves easily that $[7,8]$ :

Proposition 1. Two distributions are associated iff they are equal.

Proposition 2. The classical product of piecewise continuous functions (when considered as an element of $\mathscr{G}$ through the inclusion $\left.\mathscr{C}_{f} \subset \mathscr{G}\right)$ is associated with their product in $\mathscr{G}$.

A similar result holds for most classical multiplications of distributions, see $[3,8,22,26]$. Thus the association is an extension of the equality of distributions and through it one obtains coherence of the new product in $\mathscr{G}$ with the classical products when the latter exist.

\section{3. "SAFETY BARRIERS," OR REGULARITY RESULTS}

In order to manipulate freely one must leave the ordinary world of classical functions. Then one finds "abstract objects" that are solutions of equations. The genuine difficulty is shifted to the final task of ascertaining whether the solutions thus obtained are indeed "classical objects" which are capable of representing physical quantities. This has been done in the context of the present theory $[3,5,23-25]$. Note that for certain equations which have sufficiently many classical solutions the abstract solutions are automatically classical solutions; these results are welcome and beautiful from the mathematical viewpoint. They are all the more useful in the present setting as there are "wild objects" in $\mathscr{G}$ : for instance if $\delta$ is the Dirac distribution at the origin the "pointvalues" $\delta(0), \delta^{2}(0), \ldots$ can be considered as constant generalized 
functions (one defines pointvalues of elements of $\mathscr{G}$ as "generalized numbers" by following, for fixed $x$, the pattern of the definition of $\mathscr{G}$ ). Their classes in $\mathscr{G}$ are solutions of $G^{\prime}=0$. They are eliminated at once by classical initial or boundary conditions (which hold in a physical context); one proves easily

Proposition 3. Let $G \in \mathscr{G}$. Assume $G^{\prime}=0$ and assume there is $x_{0} \in \mathbf{R}$ such that $G\left(x_{0}\right)$ is a classical number. Then $G$ is identical to this constant.

If $G^{\prime} \approx 0$ one obtains a similar result, see [3].

In many nonlinear problems these wild constants are eliminated even without initial or boundary conditions [21]:

Theorem 1. Let $P(x, y)$ be a nonzero polynomial in two variables. Let $I$ be an open interval. Then if $G \in \mathscr{G}(I), P(x, G)=0$ if and only if $G$ is a classical $C^{\infty}$ solution on $I$.

Direct proofs of Corollaries 1, 2 and 3 are easy and given in Appendix 2.

Corollary 1. If $P$ is a nonzero polynomial in one variable, then $P(G)=0$ if and only if $G$ is identical to a classical root of $P$.

Corollary 2. The equation $x G=0, G \in \mathscr{G}$, implies $G=0$ on the whole of $\mathbf{R}$ (i.e. the singularity $x=0$ does not allow new solutions).

Corollary 3. The equation $G^{2}=x^{2}, G \in \mathscr{G}$, implies $G$ equals one of the two $C^{\infty}$ solutions $+x$ or $-x$ (the classical solutions $|x|$ and $-|x|$ are excluded). The equation $G^{4}=x^{2}$ has no solution on the whole of $\mathbf{R}$.

Note that the classical solutions $\delta, \pm|x|$ and combinations of $\pm \sqrt{|x|}$ are recovered with the association (as solutions of $x y \approx 0$, $y^{2}-x^{2} \approx 0$ and $y^{4}-x^{2} \approx 0$, respectively). From Proposition 2 :

Proposition 4. The classical continuous solutions are recovered if one states the equation in the association sense, i.e. $P(x, G) \approx 0$.

Algebraic differential equations (ADE) provide a setting in which multiplication and derivation are combined. An ADE is an equation of the form

$$
P\left(x, y(x), y^{\prime}(x), \ldots, y^{(m)}(x)\right)=0
$$

where $P$ is a nonzero polynomial in $m+2$ variables. 
Problems. Let $G \in \mathscr{G}$ and let us assume there is $x_{0} \in \mathbf{R}$ such that the pointvalues $G\left(x_{0}\right), G^{\prime}\left(x_{0}\right), \ldots, G^{(m-1)}\left(x_{0}\right)$ are classical numbers. If (4) holds in $\mathscr{G}$ with $y=G$, in which conditions is $G$ a classical solution? (See a counterexample and Corollary 4 in Appendix 2.) Is it possible to drop the condition at $x_{0}$ if $G$ is in the algebra spanned by the distributions?

The study of the nature of different kinds of solutions of ADEs in conjunction with the results exposed in [27-29] would clarify the "standard" or "nonstandard" character of these contexts; perhaps it could also clarify the concept of solution of an ADE; note that if $y$ is a function of class $C^{m}$ then $P\left(x, y(x), \ldots, y^{(m)}(x)\right) \approx 0$ in $\mathscr{G}$ if and only if $y$ is a solution in the classical sense.

\section{Generalized SOlutions of PARTIAL DifFERENTIAL EQUATIONS I: MATHEMATICS}

For large classes of equations which do not have solutions within distribution theory one can obtain existence-uniqueness results in the present setting. These new solutions are always associated with the classical solutions when the latter exist. We only give one typical example [23].

Consider the initial value problem for the semilinear hyperbolic system in two variables

$$
\begin{aligned}
\left(\partial_{t}+\Lambda(x, t) \partial_{x}\right) u(x, t) & =F(x, t, u(x, t)) \quad(x, t) \in \mathbf{R}^{2} \\
u(x, 0) & =u_{0}(x) \quad x \in \mathbf{R}^{n}
\end{aligned}
$$

$u: \mathbf{R}^{2} \rightarrow \mathbf{R}^{n}, \Lambda(x, t)$ is a smooth real-valued diagonal $n \times n$ matrix such that $\Lambda$ or $\partial_{x} \Lambda$ is globally bounded; the function $F: \mathbf{R}^{2} \times \mathbf{R}^{n} \rightarrow \mathbf{R}^{n}$ is smooth and satisfies the bounded gradient condition: for any compact subset $K$ of $\mathbf{R}^{n}$ and any $j=1, \ldots, n$

$$
\sup _{\substack{(x, t) \in K \\ u \in \mathbf{R}^{n}}}\left|\partial_{u_{j}} F(x, t, u)\right|<+\infty .
$$

Further, in order to define $F(x, t, u(x, t))$ for any $u \in\left(\mathscr{G}\left(\mathbf{R}^{2}\right)\right)^{n}$ let us assume that the map $u \rightarrow F(x, t, u)$, together with all derivatives, is polynomially bounded, uniformly for $(x, t)$ in compact subsets of $\mathbf{R}^{2}$ (remark in Appendix 1).

Theorem 2. For any given $u_{0} \in(\mathscr{G}(\mathbf{R}))^{n}$, system (5) has a unique global solution $u \in\left(\mathscr{G}\left(\mathbf{R}^{2}\right)\right)^{n}$. Moreover if $u_{0} \in\left(L_{\text {loc }}^{1}(\mathbf{R})\right)^{n}$ then 
this solution $u \in\left(\mathscr{G}\left(\mathbf{R}^{2}\right)\right)^{n}$ is associated with the classical $L_{\mathrm{loc}}^{1}\left(\mathbf{R}^{2}\right)$ solution.

Here $L_{\text {loc }}^{1}$ denotes as usual the classical space of locally integrable functions for the Lebesgue measure; such a global solution is known to exist in this setting.

One can perform explicit computations, for instance, in the case where $u$ is a distribution with support at finitely many points, see [23].

Other examples are given in $[3,5,9,12,24-26]$.

\section{GeNERALIZED SOlUtions OF PARTIAL DIFFERENTIAL EQUATIONS II: Physics}

In many domains the equations of physics put in evidence "multiplications of distributions" which follow directly from the statement of constitutive equations. In a few cases it appears impossible to avoid this fact and one really needs to deal with it. For instance Hooke's law of elasticity is a linear stress-strain relationship [20]; but in strong collisions (such as those occurring between projectiles and armour) there is not even a bijective relationship between stress and strain. In this case most physicists and engineers state Hooke's law in infinitesimal form in a frame of reference following the medium; in elastoplasticity this unavoidably gives rise to multiplications of distributions in the case of shock waves, see $[3,10,13,14,16]$.

The following is a simplified model of elasticity in a one-dimensional homogeneous medium

$$
\begin{gathered}
\rho_{t}+(\rho u)_{x}=0, \\
(\rho u)_{t}+\left(\rho u^{2}\right)_{x}=\sigma_{x}, \\
\sigma_{t}+u \sigma_{x}=k^{2} u_{x},
\end{gathered}
$$

where $\rho=$ density, $u=$ velocity, $\sigma=$ stress, $k^{2}>0$ is a constant depending on the medium and obtained from experiments (Hooke's law). Often the term $u \sigma_{x}$ is dropped in the literature (linear approximation) but for numerical simulations of collisions it plays a basic role. Numerical codes of engineers have put in evidence "solutions" $(\rho, u, \sigma)$ of (7) which are discontinuous on the same curve in the $(x, t)$ space; they represent shock waves which have been observed by physicists on the occasion of collisions. Then the term $u \sigma_{x}$ appears in the form of a product of a 
discontinuous function and a derivative of a discontinuous function, whose singularities overlap: this product does not make sense within distribution theory.

Thus one is led to formulate (7) in the present setting. One can prove that (7) has no discontinuous solution if all equations in (7) are stated with the (strong) equality in $\mathscr{G}$. On the other hand one can prove that, if one states all equations in (7) with the association, then (7) admits an infinite number of different solutions, depending on an arbitrary real parameter (arising from the term $u \sigma_{x}$ ) (see Appendix 3). This ambiguity only shows that this weak formulation does not contain enough physical information. One resolves naturally the ambiguity as follows:

The two first equations in (7) express the basic physical laws of mass and momentum conservation while the third one is a constitutive equation depending on the material and on the conditions of the experiment. A natural way to state (7) is:

$$
\begin{gathered}
\rho_{t}+\left(\rho_{u}\right)_{x}=0, \\
(\rho u)_{t}+\left(\rho u^{2}\right)_{x}=\sigma_{x}, \\
\sigma_{t}+u \sigma_{x} \approx k^{2} u_{x} .
\end{gathered}
$$

The association there expresses that, in the very small width of the shock (several times the average distance between molecules) the constitutive equation is no longer valid while mass and momentum conservation are valid there. One proves that $\left(7^{\prime}\right)$ has discontinuous solutions and nonambiguous jump conditions on the shocks; see $[3,14]$ and Appendix 3 . Our setting gives formulas, numerical methods and justifies existing numerical codes elaborated by engineers. Thus it gives the possibility to investigate problems of physics which could not be attacked mathematically within distribution theory. This method has been successfully applied to more complicated systems of elasticity, elastoplasticity, and acoustics, see $[1-3,10,11,14,16-18]$.

Thus our new concepts have permitted us to predict numerical results that emerge from experiments. In some cases it was previously unknown how to obtain them. When some data are available the results of our calculations agree qualitatively and quantitatively with the expected results $[3,10,11,13-16,18]$. Let us pause for a minute on this basic achievement, so as to understand its mechanism. The ambiguities appearing in equations of physics, when these equations involve "heuristic multiplications of distri- 
butions," correspond to the fact that these equations, when stated in weak form (i.e. with association), have an infinite number of solutions. This point was essentially known and understood without our theory (consider in quantum field theory the Hahn-Banach method of Bogoliubov-Parasiuk [4]). The basic point is that our new setting has suggested more precise formulations of the equations, on physical ground, in which there is no more ambiguity. To resolve the ambiguity physics and mathematics have been used conjointly and simultaneously, each of them playing its natural role.

6. GeNERALIZED SOlUtions OF PARTIAL DIFFERENTIAL EQUATIONS III: SYSTEMS OF CONSERVATION LAWS

A conservation law is an equation in divergence form

$$
u_{t}+\operatorname{Div}(f(u))=0 \text {. }
$$

Many physical laws are conservation laws; the more important system of conservation laws is certainly the system of fluid dynamics; in one dimension and absence of viscosity, thermal effects and external forces it is the system of equations

$$
\begin{gathered}
\rho_{t}+(\rho u)_{x}=0, \\
(\rho u)_{t}+\left(\rho u^{2}+p\right)_{x}=0, \\
(\rho e)_{t}+(\rho e u+p u)_{x}=0,
\end{gathered}
$$

where $\rho=$ density, $u=$ velocity, $p=$ pressure, and $e=$ density of total energy. Since (9) is a system of three equations with four unknowns it is complemented by a constitutive equation

$$
p=\Phi\left(\rho, e-\frac{1}{2} u^{2}\right) .
$$

All these equations are understood in the sense of distribution theory in the case of shock waves. According to the method exposed in the above section we state $(9),\left(9^{\prime}\right)$ in the more precise form

$$
\begin{gathered}
\rho_{t}+(\rho u)_{x}=0, \\
(\rho u)_{t}+\left(\rho u^{2}+p\right)_{x}=0, \\
(\rho e)_{t}+(\rho e u+p u)_{x}=0, \\
p \approx \Phi\left(\rho, e-\frac{1}{2} u^{2}\right) .
\end{gathered}
$$

It can be easily shown that $\left(9^{\prime \prime}\right)$ has travelling wave solutions (i.e. solutions which remain constant on both sides of the discontinuity 
and propagate with constant speed) and that they are solutions of the system of equations in nonconservation form

$$
\begin{gathered}
v_{t}+u v_{x}-v u_{x}=0 \\
u_{t}+u u_{x}+v p_{x}=0 \\
\left((v)^{-1} \frac{\partial \varphi}{\partial p}(v, p)\right)\left(p_{t}+u p_{x}\right)+\left(p+\frac{\partial \varphi}{\partial p}(v, p)\right) u_{x} \approx 0,
\end{gathered}
$$

where $v=1 / \rho$ is the specific volume and where the constitutive equation has been stated in the form $e-\frac{1}{2} u^{2} \approx \varphi(v, p)$. Note that (10) has no discontinuous solution in the sense of distribution theory, but has discontinuous solutions in our setting. Travelling wave solutions of (10) have a very simple form $[3,14]$ and, from this study, one can build numerical schemes for the solution of (10) $[3,13,15,18]$. In certain circumstances this method is very efficient (modelling of the behavior of solids submitted to strong constraints).

Note also that solutions in $\mathscr{G}$ of systems of conservation laws are very closely connected to the measure valued solutions of Di Perna [19].

\section{ConClusion}

In conclusion one can stress the basic role played by the dissociation of the classical concept of equality into the strong equality in $\mathscr{G}$ and into the weak equality $\approx$. This dissociation permits one to circumvent Schwartz's impossibility result, and so to obtain a general multiplication of distributions (enjoying all computational properties) coherent with classical analysis. From a viewpoint of applied mathematics and physics it is at the very basis of the resolution of ambiguities and thus of the predictions of results that could be checked from experiments. A posteriori one can realize that this dissociation had already been perceived, in the very classical setting of ADEs by Rubel [27-29] in his dissociation of solutions of ADEs into $C^{\infty}$ ones and "pointwise" ones (i.e. only differentiable enough to plug into the $\mathrm{ADE}$ ). We can also retain that the theory presented in this paper can be considered as some kind of "nonstandard analysis" since it realizes a calculus involving "infinitesimal quantities." There the concept of "shadow" of a nonstandard function might play the role of our association. An up to date set of references can be found in the second edition of [3]. 


\section{APPENDIX 1. DEFINITION OF GENERALIZED FUNCTIONS}

If $q=0,1,2, \ldots$ we set

$$
\begin{aligned}
& \mathscr{A}_{q}=\left\{\varphi \in \mathscr{D} \text { such that } \int_{-\infty}^{+\infty} \varphi(\lambda) d \lambda=1\right. \text { and } \\
& \left.\qquad \int_{-\infty}^{+\infty} \lambda^{i} \varphi(\lambda) d \lambda=0 \text { if } 1 \leq i \leq q\right\} .
\end{aligned}
$$

One proves easily that $\mathscr{A}_{q}$ is nonvoid; if $\varphi \in \mathscr{D}$ and $\varepsilon>0$ we set

$$
\varphi_{\varepsilon}(\lambda)=\frac{1}{\varepsilon} \varphi\left(\frac{\lambda}{\varepsilon}\right)
$$

Then it is immediate that $\varphi \in \mathscr{A}_{q}$ if and only if $\varphi_{\varepsilon} \in \mathscr{A}_{q}$. We denote by $\mathscr{E}_{0}$ the set of all functions

$$
\begin{aligned}
F: \mathscr{A}_{0} \times \mathbf{R} \rightarrow \mathbf{R} \\
\varphi, x \quad F(\varphi, x)
\end{aligned}
$$

such that for every $\psi, \chi \in \mathscr{A}_{0}$ the map

$$
\underset{\theta}{\mathbf{R}} \times] 0, \underset{\varepsilon}{1}\left[\times \underset{x}{\mathbf{R}} \rightarrow \underset{F}{\mathbf{R}}\left([\theta \psi+(1-\theta) \chi]_{\varepsilon}, x\right)\right.
$$

is $C^{\infty}$ in the variables $(\theta, \varepsilon, x)$. We denote by $\mathscr{E}_{M}$ (where the subscript $M$ stands for moderate) the subset of $\mathscr{E}_{0}$ of all functions $F$ such that $\forall n, m \in \mathbf{N} \quad \exists N \in \mathbf{N}$ such that if $\varphi \in \mathscr{A}_{N}$ there are (*) $c, \eta>0$ such that

$$
\sup _{|x| \leq n}\left|\frac{d^{m}}{d x^{m}} F\left(\varphi_{\varepsilon}, x\right)\right| \leq c \varepsilon^{-N} \quad \text { if } 0<\varepsilon<\eta .
$$

The symbol $(*)$ always means that $c$ and $\eta$ can be chosen independent of $\varphi$ when $\varphi$ ranges in a closed line segment in the set $\mathscr{A}_{N}$ (i.e. $\left.\varphi=\theta \psi+(1-\theta) \chi, \psi, \chi \in \mathscr{A}_{N}, 0 \leq \theta \leq 1\right)$. Clearly $\mathscr{E}_{M}$ is a subalgebra of $\mathscr{E}_{0}$ (for pointwise multiplication). We denote by $\mathscr{N}$ the set of all functions $F \in \mathscr{E}_{0}$ such that

$\forall n, m \in \mathbf{N} \forall p \in \mathbf{N} \exists q \in \mathbf{N}$ such that if $\varphi \in \mathscr{A}_{q}$ there are (*) $c, \eta>0$ such that

$$
\sup _{|x| \leq n}\left|\frac{d^{m}}{d x^{m}} F\left(\varphi_{\varepsilon}, x\right)\right| \leq c \varepsilon^{p} \quad \text { if } 0<\varepsilon<\eta .
$$

Clearly $\mathscr{N}$ is an ideal of $\mathscr{E}_{M}$ and so the quotient space

$$
\mathscr{G}=\mathscr{E}_{M} / \mathscr{N}
$$


is an algebra. It is proved that the elements of $\mathscr{G}$ have essentially the properties of $C^{\infty}$ functions (they are local objects, derivation, multiplication, ... ) see $[3,9,26]$.

Remark. The bounds defining $\mathscr{N}$ came from the idea of extension of the ideal Ker $\mathfrak{M}$ of $\mathscr{C}^{\infty}\left(\mathscr{C}^{\prime}\right)$; then the bounds defining $\mathscr{E}_{M}$ came from the idea of finding a subalgebra of $\mathscr{C}^{\infty}(\mathscr{D})$ for which $\mathscr{N}$ would be an ideal, see [7,8].

To $g \in \mathscr{C}^{\infty}$ we associate $R \in \mathscr{E}_{M}$ defined by $R(\varphi, x)=g(x)$. One shows easily that this defines an inclusion $\mathscr{C}^{\infty} \subset \mathscr{G}$.

Let us denote by $\mathscr{C}_{f}$ the set of all piecewise continuous functions on $\mathbf{R}$ (i.e. continuous except on a discrete set, on which they have right and left limits). To $g \in \mathscr{C}_{f}$ we associate $R \in \mathscr{E}_{0}$ defined by

$$
R(\varphi, x)=\int_{-\infty}^{+\infty} g(\lambda) \varphi(\lambda-x) d \lambda .
$$

One proves at once that $R \in \mathscr{E}_{M}$ and that this defines an inclusion $\mathscr{C}_{f} \subset \mathscr{G}$. If $g \in \mathscr{C}^{\infty}$ one checks at once that the choice between the two above-mentioned functions $R$ is insignificant modulo $\mathscr{N}$. If $T$ is a distribution one defines $R$ by an immediate extension of the above formula and one obtains an inclusion of the set $\mathscr{D}^{\prime}$ of all distributions into $\mathscr{G}$. If one does not know the distributions one may define them as those elements of $\mathscr{G}$ which are, in the neighborhood of each real number, some derivative of a continuous function.

Remarks. In $[7,8]$ we gave a slightly different definition of the ideal $\mathscr{N}$; it has been recognized subsequently [9] that the definition given above is necessary to provide uniqueness of asymptotic expansions, uniqueness of analytic continuation, and also Theorem 1 below.

In [9] the differentiability of $F$ in $\theta$ and $\varepsilon$, which is present in $[7,8]$, was dropped for simplification. It was recognized later that this condition is needed to obtain Theorem 1.

More generally than multiplication, one can define $f(G), G \in$ $\mathscr{G}\left(\mathbf{R}^{n}\right)$ arbitrary, provided that $f$ is a $C^{\infty}$ function which, together with all its derivatives, is polynomially bounded.

The concept of equality in $\mathscr{G}$ is very strict. We introduce the weaker concept of "association": an element $G$ of $\mathscr{G}$ is said to be associated with 0 iff for any $\psi \in \mathscr{C}_{0}^{\infty}(\Omega)$ the integral

$$
\int_{\mathbf{R}} R\left(\varphi_{\varepsilon}, x\right) \psi(x) d x
$$


tends to 0 when $\varepsilon \rightarrow 0$ ( $R$ is a representative of $G, \varphi$ is in $\mathscr{A}_{N}$ for large enough $N$ ).

We say that $G_{i}$ and $G_{2} \in \mathscr{G}$ are associated with each other iff $G_{1}-G_{2}$ is associated with 0 ; we write $G_{1} \approx G_{2}$. The concept of association is a faithful generalization of the classical concept of equality of integrable functions and distributions. But it is through our concept of equality in $\mathscr{G}$ that we are able to define the product of distributions.

\section{APPENDIX 2. Proofs of Regularity ResUlts}

Direct proof of Corollary 1. Let $a_{1}, a_{2}, \ldots, a_{n}$ be the classical roots of $P, a_{i} \neq a_{j}$ if $i \neq j$. By assumption we have a bound $\left|P\left(R\left(\varphi_{\varepsilon}, x\right)\right)\right| \leq c_{q} \varepsilon^{\alpha(q)}$ if $R$ is a representative of $G(\alpha(q)$ tends to $+\infty$ when $q$ tends to $+\infty)$. Taking into account the multiplicity of the roots of $P$ we get

$$
\left|R\left(\varphi_{\varepsilon}, x\right)-a_{i_{0}}\right| \leq c_{q}^{\prime} \varepsilon^{\alpha^{\prime}(q)} \quad \text { for some other } c_{q}^{\prime}, \alpha^{\prime},
$$

where $a_{i_{0}}$ depends $a$ priori on $\varepsilon, \varphi$ and $x$; but from the continuity of $R\left(\varphi_{\varepsilon}, x\right)$ in $\varepsilon, \varphi$ and $x$ one has $a_{i_{0}}$ independent of $\varepsilon, \varphi$ and $x$. It remains necessary to obtain a bound for the $x$ derivatives of $R\left(\varphi_{\varepsilon}, x\right)$. One has

$$
\frac{d}{d x} P\left(R\left(\varphi_{\varepsilon}, x\right)\right)=P^{\prime}\left(R\left(\varphi_{\varepsilon}, x\right)\right) \cdot R^{\prime}\left(\varphi_{\varepsilon}, x\right)
$$

so that if $P^{\prime}\left(a_{i_{0}}\right) \neq 0$ one has at once the required bound for $\left|R^{\prime}\left(\varphi_{\varepsilon}, x\right)\right|$. If $P^{\prime}\left(a_{i_{0}}\right)=0$ and if $P^{\prime \prime}\left(a_{i_{0}}\right) \neq 0$, the formula

$$
\begin{aligned}
\frac{d^{2}}{d x^{2}} P\left(R\left(\varphi_{\varepsilon}, x\right)\right)= & P^{\prime \prime}\left(R\left(\varphi_{\varepsilon}, x\right)\right) \cdot\left(R^{\prime}\left(\varphi_{\varepsilon}, x\right)\right)^{2} \\
& +P^{\prime}\left(R\left(\varphi_{\varepsilon}, x\right)\right) \cdot R^{\prime \prime}\left(\varphi_{\varepsilon}, x\right)
\end{aligned}
$$

gives at once the required bound for $\left|R^{\prime}\left(\varphi_{\varepsilon}, x\right)\right|$ using (11) to get rid of the last term. By induction on the order of multiplicity of $a_{i_{0}}$, we get a bound $\mid R^{\prime}\left(\varphi_{\varepsilon}, x \mid \leq c_{q}^{\prime \prime} \varepsilon^{\alpha^{\prime \prime}(q)}\right.$. Similarly one gets bounds for higher-order derivatives of $R$.

Direct proof of Corollary 2. $x y=0 \Rightarrow x y^{\prime}+y=0 \Rightarrow x^{2} y^{\prime}=0 \Rightarrow$ $x^{2}\left(y^{\prime}\right)^{3}=0$. Let $R\left(\varphi_{\varepsilon}, x\right)$ be a representative of $y$. In abbreviated notation we have $\left|x^{2} R^{\prime 3}\left(\varphi_{\varepsilon}, x\right)\right| \leq c_{q}^{\prime} \varepsilon^{\alpha^{\prime}(q)}$. Since $|x|^{-2 / 3}$ is integrable at 0 one finds that $\left|R\left(\varphi_{\varepsilon}, x\right)\right| \leq c_{q}^{\prime} \varepsilon^{\alpha^{\prime}(q)}$ for some 
other $c_{q}^{\prime}, \alpha^{\prime}$ (if $x \geq 0$ use the formula $R\left(\varphi_{\varepsilon}, x\right)=R\left(\varphi_{\varepsilon}, 1\right)+$ $\int_{1}^{x} R^{\prime}\left(\varphi_{\varepsilon}, \lambda\right) d \lambda$; if $x \leq 0$, replace 1 by -1$)$. To get a similar bound for $R^{\prime}\left(\varphi_{\varepsilon}, x\right)$ start from $x^{2} y^{\prime}=0$; setting $z=y^{\prime}$ one obtains as at the beginning of this proof that $x^{3} z^{\prime 4}=0$. One concludes from the fact that $|x|^{-3 / 4}$ is integrable at $0 . x^{2} y^{\prime}=0 \Rightarrow$ $2 x y^{\prime}+x^{2} y^{\prime \prime}=0 \Rightarrow x^{3} y^{\prime \prime}=0$, from which one can use the same method to get the desired bound for $R^{\prime \prime}$. And so on.

Direct proof of Corollary 3. $y^{2}=x^{2}$ in $\mathscr{G}(\mathbf{R})$ implies $y y^{\prime}=x$, $y^{2} y^{\prime}=y x$ and so $x^{2} y^{\prime}=x y$, i.e. $x\left(x y^{\prime}-y\right)=0$. From Corollary $3, y=x y^{\prime}$. This equality and $(y-x)(y+x)=0$ imply $x^{2}\left(y^{\prime}-1\right) \times$ $\left(y^{\prime}+1\right)=0$. Corollary 3 gives $\left(y^{\prime}-1\right)\left(y^{\prime}+1\right)=0$. Corollary 2 gives that $y^{\prime}$ is identical to the constant +1 or to the constant -1 . Integration (Proposition 3) gives $y=x+c_{1}$ or $y=-x+c_{2}$, where $c_{1}, c_{2}$ are generalized numbers. From $y^{2}=x^{2}$ one gets $c_{1}=0=c_{2}$.

Another consequence of Theorem 1 is:

Corollary 4. Under the conditions on $P$ and $I$ in Theorem 1 let us consider the $\operatorname{ADE} P\left(x, y^{(m)}(x)\right)=0$ in $\mathscr{G}$, completed by the condition

$$
\begin{aligned}
& y\left(x_{0}\right)=\alpha_{0}, \ldots, y^{(m-1)}\left(x_{0}\right)=\alpha_{m-1}, \\
& \alpha_{i} \in \mathbf{R}, \quad 1 \leq i \leq m-1 \text { and } x_{0} \in I .
\end{aligned}
$$

Then $P\left(x, G^{(m-1)}\right)=0$ in $\mathscr{G}(I)$ iff $G$ is a classical $C^{\infty}$ solution. Proof. Apply Theorem 1 with $G^{(m)}$ and integrate (Proposition 3).

Counterexample. The class of $R(\varphi, x)=f(\varphi) \exp \left(1 /\left(x^{2}-1\right)\right)$ if $-1 \leq x \leq 1$ and $R(\varphi, x)=0$ if $x \leq-1$ or $x \geq 1$, where $f(\varphi)=\int \varphi^{2}(\lambda) d \lambda$, is solution of the equation $2 x y+\left(x^{2}-1\right) y^{\prime}=0$.

\section{APPENDiX 3. EXamples of SHOCK WAVE Calculations}

We sketch how one can compute shock wave solutions of system $\left(7^{\prime}\right)$. First consider the weak formulation

$$
\begin{gathered}
\rho_{t}+(\rho u)_{x} \approx 0, \\
(\rho u)_{t}+\left(\rho u^{2}\right)_{x} \approx \sigma_{x}, \\
\sigma_{t}+u \sigma_{x} \approx k^{2} u_{x} .
\end{gathered}
$$


We seek travelling wave solutions of the form

$$
\begin{aligned}
& \rho(x, t)=\Delta \rho H(x-v t)+\rho_{1}, \\
& u(x, t)=\Delta u K(x-v t)+u_{1}, \\
& \sigma(x, t)=\Delta \sigma L(x-v t)+\sigma_{1},
\end{aligned}
$$

where $v$ (= velocity of the shock), $\Delta w=w_{r}-w_{1}, w(w=$ $\rho, u, \sigma)$ are real numbers and where $H, K, L$ are three possibly different Heaviside generalized functions (i.e. they are associated with the classical Heaviside function). Putting (12) into $\left(7^{\prime \prime}\right)$ one obtains at once the three jump formulas

$$
\begin{gathered}
\left(v-u_{1}\right) \Delta \rho=\left(\Delta \rho+\rho_{1}\right) \Delta u, \\
\left(v-u_{1}-\Delta u\right)\left(\Delta \rho \Delta u+\rho_{1} \Delta u+u_{1} \Delta \rho\right)=u_{1} \rho_{1} \Delta u-\Delta \sigma, \\
\left(v-u_{1}\right) \Delta \sigma=A \Delta u \Delta \sigma-k^{2} \Delta u,
\end{gathered}
$$

where the real parameter $A$ is defined by $K L^{\prime} \approx A \delta$. There is an infinite number of possible jump conditions depending on the real parameter $A$.

Now let us consider the stronger formulation $\left(7^{\prime}\right)$. The first equation in $\left(7^{\prime}\right)$ gives

$$
\left(-v+u_{1}+\Delta u K\right) H^{\prime}+\rho_{1} \frac{\Delta u}{\Delta \rho} K^{\prime} H+\rho_{1} \frac{\Delta u}{\Delta \rho} K^{\prime}=0
$$

from which one obtains easily $H$ as a function of $K$ by solving as usual the differential equation $a(x) y^{\prime}+b(x) y+c(x)=0$ in $\mathscr{G}$. The second equation in $\left(7^{\prime}\right)$ gives $L$ as a function of $H$ and $K$. Finally one obtains $L=K$ and thus $K L^{\prime}=K K^{\prime} \approx \frac{1}{2} K^{\prime}$ (by differentiation of $K^{2} \approx K$ ), i.e. $A=\frac{1}{2}$. ( $\left.7^{\prime \prime}\right)$ has nonambiguous jump conditions. In general one finds values of numbers like $A$ which are different from $\frac{1}{2}$. Various examples of this kind of calculations are given in $[3,10,11,14,16,18]$.

\section{APPENDIX 4. DistRibutions IN MATHEMATICS AND IN PHYSICS}

We have a canonical inclusion $\mathscr{D}^{\prime} \subset \mathscr{G}$ and, at the same time, one is forced in physical applications to consider several Heaviside like, Dirac like,... functions. There is no paradox if one thinks about the different ways mathematicians and physicists conceive and use distributions.

For mathematicians the space $\mathscr{D}^{\prime}$ is defined modulo an isomorphism (concerning all operations). It is such an isomorphic copy of $\mathscr{D}^{\prime}$ which is canonically included into $\mathscr{G}$. If permits, via 
the multiplication in $\mathscr{G}$ and the association, a synthesis of most existing multiplications of distributions.

For physicists the space $\mathscr{D}^{\prime}$ is considered as a reservoir of mathematical objects used to describe the physical world. In our context the use of the above subspace $\mathscr{D}^{\prime}$ of $\mathscr{G}$ as such a reservoir may lead to mistakes in some cases involving "multiplications of distributions." Then the correct reservoir is $\mathscr{G}$ itself, which contains several Heaviside like, Dirac like,... functions.

In this way a nonambiguous mathematical multiplication of distributions can be reconciled with the well-known fact that in physics "multiplications of distributions" such as $Y \delta$ or $\delta^{2}$, can give different results according to the context. Distributions originating in physics have to be represented by various elements of $\mathscr{G}$, usually not those in the subspace $\mathscr{D}^{\prime}$ of $\mathscr{G}$. This reminds one of the nonbijective correspondence between quasi-standard functions (a subclass of the nonstandard functions) and distributions. This suggests that our theory could be considered as some refined (with respect to certain properties) version of nonstandard analysis.

\section{ACKNOWLEDGMENTS}

The author is very much indebted to an unknown referee for suggesting an overall change in the text, and to Dr. J. T. Donohue for corrections and clarifications.

\section{REFERENCES}

1. M. Adamczewski, J. F. Colombeau, and A. Y. Le Roux, Convergence of numerical schemes involving powers of the Dirac delta function, J. Math. Anal. Appl. 195 (1) 1990, 172-185.

2. A. Y. Barka, J. F. Colombeau, and B. Perrot, A numerical modeling of the fluid/fluid acoustic dioptra, J. Acoustique (in press), 1989.

3. H. A. Biagioni, Introduction to a nonlinear theory of generalized functions, Notas de Matematica, IMECC, UNICAMP, 13100, Campinas, SP Brazil, 1988. Second edition: A nonlinear theory of generalized functions, Lecture Notes in Math., vol. 1421, Springer-Verlag, Berlin, Heidelberg, New York, 1990.

4. N. N. Bogoliubov and D. V. Shirkov, Introduction to the theory of quantized fields, Interscience (various editions).

5. J. J. Cauret, J. F. Colombeau, and A. Y. Le Roux, Discontinuous generalized solutions of nonlinear nonconservative hyperbolic equations, J. Math. Anal. Appl. 139 (2) (1989), 552-573.

6. J. F. Colombeau, Differential calculus and holomorphy: Real and complex analysis in locally convex spaces, North-Holland, Amsterdam, 1982.

7. __ A multiplication of distributions, J. Math. Anal. Appl. 94 (1) (1983), 96-115. 
8. __ New generalized functions and multiplication of distributions, NorthHolland, Amsterdam, 1984.

9. __ Elementary introduction to new generalized functions, North-Holland, Amsterdam, 1985.

10. _ The elastoplastic shock problem as an example of the resolution of ambiguities in the multiplication of distributions, J. Math. Phys. 30 (10) (1989), 2273-2279.

11. __ A method to obtain correct jump conditions from systems in nonconservative form: new formulas and new numerical schemes, Num. Appl. Math. (in press).

12. J. F. Colombeau and M. Langlais, An existence-uniqueness result for a nonlinear parabolic equation with Cauchy data distribution, J. Math. Anal. Appl. 145 (1) (1990), 186-196.

13. J. F. Colombeau and A. Y. Le Roux, Numerical techniques in elastodynamics, Lecture Notes in Math., vol. 1270, Springer-Verlag, Berlin and New York, 1986, pp. 104-114.

14. _ Multiplications of distributions in elasticity and elastoplasticity, J. Math. Physics. 29 (2) (1988), 315-319.

15. __ Numerical methods for hyperbolic systems in nonconservative form using products of distributions, Advances for computer methods in PDE 6 IMACS, 1987, pp. 28-37. Far better results can be found in: P. DeLuca Modelisation numérique en elastoplasticité dynamique, thesè, Bordeaux, 1989.

16. __ Generalized functions and products appearing in equations of engineering and physics, preprint.

17. J. F. Colombeau, A. Y. Le Roux, A. Noussair, and B. Perrot, Microscopic profile of shock waves and ambiguities in multiplications of distributions, SIAM J. Numer. Anal. 26 (4) (1989), 871-882.

18. J. F. Colombeau and B. Perrot, A numerical method for the solution of nonlinear systems of physics involving multiplications of distributions, preprint.

19. R. Di Perna, Measure valued solutions to conservation laws, Arch. Rational Mech. Anal. 88 (1985), pp. 223-270.

20. Y. C. Fung, $A$ first course in continuum mechanics, Prentice Hall, Englewood Cliffs, NJ, 1969.

21. A. Marzouk and B. Perrot, Regularity results for generalized polutions of algebraic equations and algebraic differential equations, preprint.

22. M. Oberguggenberger, Products of distributions, J. Reine Angew. Math. 365 (1986), 1-11.

23. __ Generalized solutions to semilinear hyperbolic systems, Monatshe. Math. 103 (1987), 133-144.

24. __ Hyperbolic systems with discontinuous coefficients: generalized solution and a transmission problem in acoustics, J. Math. Anal. Appl. 42 (2) (1989), 452-467.

25. __ Hyperbolic systems with discontinuous coefficients: examples, Generalized Functions, Convergence Structure and Applications (B. Stankovic, E. Pap, S. Pilipovic, V. S. Vladimirov, eds.), Plenum, New York and London, 1988, pp. 257-266.

26. E. E. Rosinger, Generalized solutions to Nonlinear PDE, North-Holland, Amsterdam, 1987. 
27. L. A. Rubel, Solutions of algebraic differential equations, J. Differential Equations 49 (1983), 441-452.

28. L. A. Rubel, Some research problems about algebraic differential equations, Trans. Amer. Math. Soc. 280 (1983), 43-52.

29. __ Generalized solutions of algebraic differential equations, J. Differential Equations 62 (1986), 242-251.

Ecole Normale Supérieure de lyon, 46 Allées d' Italié, 69364 lyon Cedex 07, France 